African Journal of Biomedical Research, Vol. 8 (2005); 41 - 45

ISSN 1119-5096 (c) Ibadan Biomedical Communications Group

Available online at $\underline{h t t p: / / w w w . b i o l i n e . o r g . b r / m d ~}$

\title{
Full Length Research Article \\ Volumetric Changes in the Components of Left Ventricle Myocardium of Spontaneously Hypertensive Rats Following Exercise and ACE Inhibitor Treatment
}

\author{
'INUWA IBRAHIM M, ${ }^{2}$ HASSAN M.O, AND ${ }^{2}$ ZIADA AMAL \\ Departments of ${ }^{1}$ Human / Clinical Anatomy and ${ }^{2}$ Physiology, Sultan Qaboos \\ University, Oman.
}

Received: September, 2004

Accepted: January, 2005

\begin{abstract}
This study was designed to test the possible effects of a combination of physical and pharmacological therapy intervention on myocardial structure of the left ventricle in spontaneously hypertensive rats (SHR). Forty 12 weeks old spontaneously hypertensive rats were divided into four groups of sedentary, Sed as controls, exercise only , (Exer), lisinopril only $20 \mathrm{mg} / \mathrm{kg} / \mathrm{day}$, (Lis), and exercise + lisinopril, (LisExer). Exercise training was performed on a treadmill $(5 \mathrm{~m} / \mathrm{min}$.) for 60 minutes/day, 5 days/week for 10 weeks. At the end of 10 weeks, all the rats were terminally anaesthetised, the heart was arrested in diastole by intravenous procaine and whole animal perfusion fixation through the abdominal aorta was carried out using Karnovsky's fixative (Ph 7.24). The heart was removed and left ventricle plus the interventricular septum was serially sectioned at a thickness of three millimetres. One piece was randomly chosen, and embedded in JB4 resin. Six sections were obtained from each block, stained with toluidine blue:acid fucin. Measurement of volume fraction $V_{f}$, of myocardial components was carried out using Histometrix MIL6 Ver 1.0 stereology software (Kinetic imaging Ltd.). Mean volume fraction, $V_{f}$ of capillaries in Sed. group was $0.114 \pm 0.01$ (SEM). This was significantly increased in LisExergroup. The volume fraction of muscle in Sed group was $0.795 \pm 0.02$ (SEM). This was significantly decreased in Lis but unchanged in Exer group. Capillaries $V_{f}$ was significantly higher in LisExer as compared to Lis or Exergroups $(\mathrm{p}<0.05)$. Muscle $V_{f}$ was not different between LisExer and Lis groups. The outcome of these changes could well be a better enhancement of cardiac performance in hypertension by combined exercise and ACE inhibitor treatment than either of the interventions alone.
\end{abstract}

Keywords:

hypertrophy,ACE-inhibitor, volume fraction, exercise

\section{INTRODUCTION}

One of the non- pharmacological interventions used in the management of hypertension is moderate physical exercise. Moderate exercise is considered as a useful tool in reducing blood pressure and is often prescribed to hypertensive patients in addition to drug treatment (Mancia and Grassi 1998). Some studies indicated that moderate exercise increase left ventricular hypertrophy in rats and that the beneficial effect of exercise on hypertension is through the improvement in coronary micro vasculature (Anversa, et al. 1983; Crisman, et al. 1985).
Others have suggested that moderate exercise in humans reduces left ventricular hypertrophy (Himeno, et al. 1996; Hinderliter, et al. 2002). Moderate exercise has been shown to also induced myocardial capillary growth in the spontaneously hypertensive rat (Crisman et al. 1985).

The renin-angiotensin-aldosterone system (RAAS) plays a key role in the pathogenesis of certain types of hypertensive heart disease. Blockade of this system results in a number of biologically important beneficial effects including reduction in blood pressure as well as reversal of endothelial dysfunction. Treatment with ACE 
inhibitors reduce cardiac hypertrophy and improve coronary vascular reserve in left and right ventricles in hypertensive rats. It is believed that such drugs are able to reduce myocardial hypertrophy by inducing selective apoptosis of cardiomyocytes and interstitial fibroblasts .

One of the approaches of evaluating benefit of antihypertensive management is the assessment of reduction of myocardial hypertrophy. In humans, assessment of myocardial hypertrophy is usually done with the help of echocardiography.

An animal model of hypertension - the spontaneously hypertensive rats (SHR) is available where hypertension gets established by the age of 12 weeks. The main approach to the design of this experiment is that after experimental interventions in such animals the whole left ventricle can be obtained and by using unbiased stereology the volumes of various components of the myocardium can be obtained.

This study aims at evaluating the effect of both interventions of moderate exercise and ACE inhibitor treatment on left ventricular myocardium structure in spontaneously hypertensive rats. The hypothesis is that if ACE inhibitor treatment attenuates left ventricular hypertrophy and exercise improves ventricular vascular bed, then combining the two would lead to a better outcome with respect to reduction in myocardial hypertrophy and increased capillary volume. This could presumably improve myocardial performance better than either intervention alone.

\section{MATERIALS AND METHODS}

\section{Experimental design}

All procedures were in accordance with the principles for use of animals in research and education and approved by the medical research and ethics committee of the college of medicine (MREC). Forty-eight spontaneously hypertensive rats (SHR) were kept in pairs and housed in a temperature controlled room $\left(23-25^{\circ} \mathrm{C}\right)$ with a 12 hour day/night cycle. Food and water were available ad libitum until when they were 12 weeks old and systemic hypertension well established (average tail cuff systolic blood pressure recording was $205 \pm 5 \mathrm{mmHg}$ ).

At 12 weeks of age, the rats were andomly divided into four groups $(\mathrm{n}=10)$; sedentary, Sed. as controls, exercise only, Exer, $20 \mathrm{mg} / \mathrm{kg} /$ day lisinopril only, Lis, and combined exercise + lisinopril, LisExer. Exercise training was performed on a motor treadmill $(5 \mathrm{~m} / \mathrm{min}$.) for 60 minutes/day, 5 days/week for 12 weeks. Lisinopril, an ACE inhibitor was administered daily intraperitoneally for 12 weeks.

\section{Animal sacrifice and obtaining tissues}

At the end of 12 weeks, each animal was weighed before induction of anesthesia. Thereafter, intraperitoneal injection of sodium pentobarbitone $(50 \mathrm{mg} / \mathrm{kg})$ was given. The abdomen was opened via a midline incision. Abdominal aorta and inferior vena cava were identified and canulated. Whilst the heart was still beating 1000units of heparin was injected through the IVC to prevent blood clotting. After one minute of heparin injection, one milliliter of $2 \%$ procaine was injected also through the IVC, which stopped the heart beating in diastole. Through the aortic canula, $100 \mathrm{ml}$ of Karnovski's fixative was injected at a pressure of $160 \mathrm{mmHg}$ until the heart was fully perfused. Thereafter, the whole heart was removed weighed, and immersed in the same fixative for one hour. The left ventricle was excised weighed and serially sectioned into $3 \mathrm{~mm}$ pieces in a "small object slicer" filled with molten paraffin wax.

\section{Estimation of volume fractions $V_{f}$}

One slice of the ventricle was cut using the orientator method (Mattfeldt et al. 1990) so as to obtain an isotropic uniform random tissue face. This tissue face was embedded in JB4 resin and six $1.5 \mu \mathrm{m}$ sections obtained on a microtome (Leica RM2145). The sections were stained with combined toluidine blue:acid fucsin (muscle appear blue while capillaries appear red). From each section, ten systematic random fields were sampled and estimaes of volume fractions of capillaries, myocardium, and interstitial tissue obtained. This was carried out with the help of a stereology software (Histometrix MIL6 Ver 1.0, Kinetic Imaging Ltd. England) installed on an IBM personal computer.

All data was presented as mean \pm standard error of mean. Analysis of the data was by the use of analysis of variance (ANOVA) followed by Student-Newman-Keuls Multiple Comparisons post-hoc test. The selected criterion for statistical significance was when the two-tailed $p$ value was less than 0.05

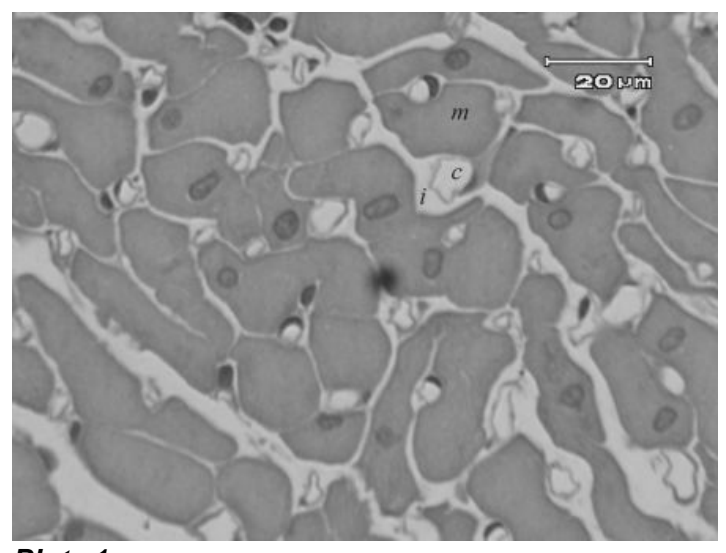

Plate 1

Photomicograph of left ventricle myocardium in SHR. capillaries (c), muscle (m), interstitium (i). 


\section{RESULTS}

Mean volume fraction, $V_{f}$ of capillaries in Sed. group was $0.114 \pm 0.01$ (SEM). This was increased by $7 \% \quad(p<0.01), 6 \% \quad(p<0.01)$ and $12 \%(p<0.001)$ in the Lis, Exer, and LisExer groups respectively. The volume fraction of muscle in Sed. group was $0.795 \pm 0.02$ (SEM). This has decreased $11.4 \% \quad(p<0.01), 3.3 \%$ $(p>0.05)$, and $16.5 \%(p<0.01)$ in the Lis, Exer, and LisExer groups respectively. Volume fraction of interstitial tissue was $0.106 \pm 0.02$ (SEM) in the Sed. rats. This has decreased $3.8 \%$, and $3.9 \%$ in Lis, and LisExer groups respectively $(p>0.05)$. It was unchanged in the Exer group (Fig 1 a-c).

(a) muscle

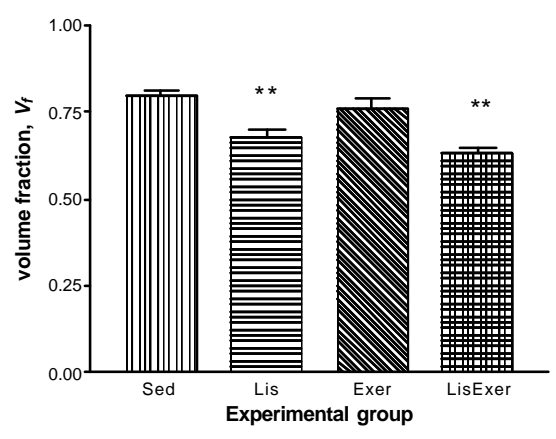

(b) interstitium

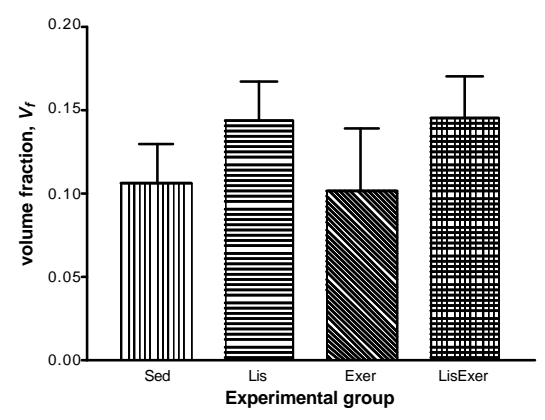

(c) capillaries

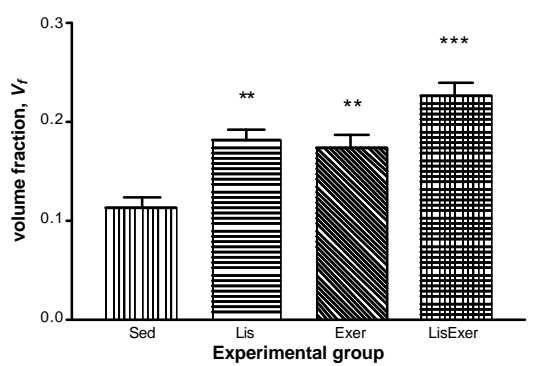

Figure 1

Mean volume fraction of myocardium components in Sed, Lis, Exer, and LisExer groups. (a) muscle, (b) interstitium, (c) capillaries. ${ }^{\star \star} p<0.01,{ }^{\star \star \star} p<0.001$

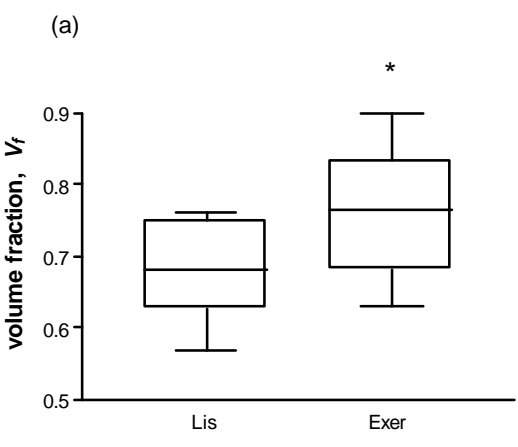

(b)

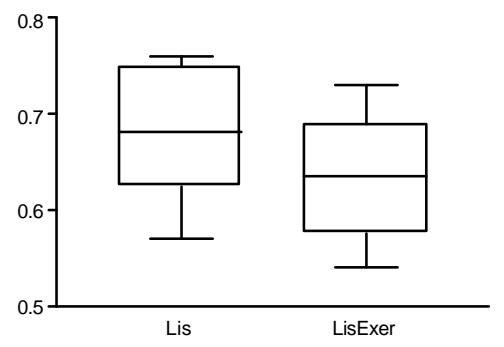

(c)

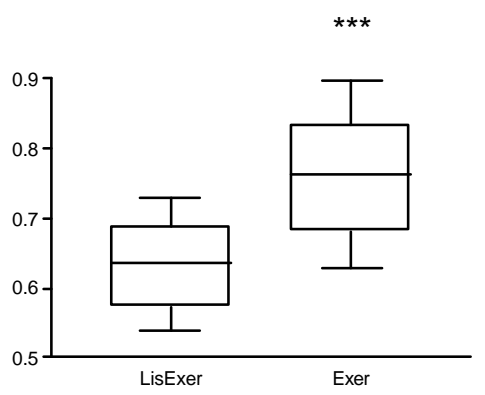

Figure 2

Box plot comparison of $V_{f}$ muscle between intervention groups. (a) between Lis and Exer, (b) between Lis and LisExer, (c) between LisExerand Exer.

${ }^{\star} p<0.05,{ }^{* *} p<0.01,{ }^{\star \star *} p<0.001$

There was no significant difference in $V_{f}$ of capillaries between Lis and Exergroup. LisExer had significantly higher $V_{f}$ of capillaries as compared to Lis and Exer groups $(\mathrm{p}<0.05)$ (Fig. 4 a-c).

Lis group had significantly smaller $V_{f}$ of muscle when compared to Exer group $(p<0.05)$. In LisExer group, $V_{f}$ of muscle was significantly smaller when compared to Exer group $(p<0.001)$, although there was no significant difference when compared to Lis group (Fig 3 a c). Volume fraction of interstitial tissue was not significantly different $(p>0.05)$ between all experimental groups (Fig $3 \mathrm{a}-\mathrm{c}$ ). 
(a)

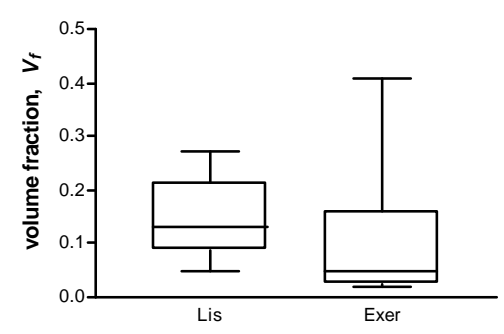

(b)

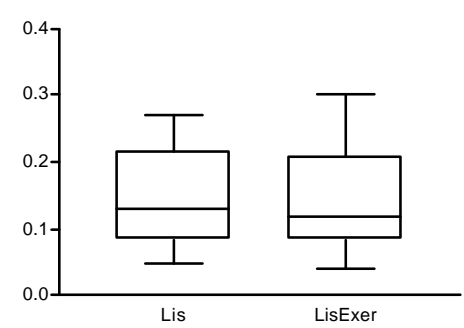

(c)

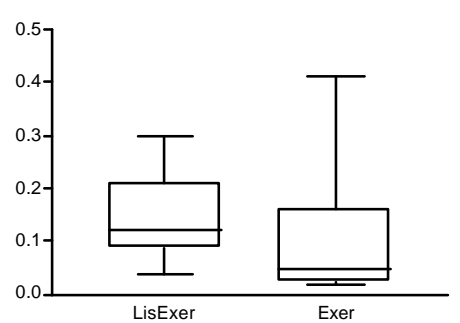

Figure 3

Box plot comparison of $V_{f}$ interstitium between intervention groups. (a) between Lis and Exer, (b) between Lis and LisExer, (c) between LisExer and Exer.

${ }^{\star} \mathrm{p}<0.05,{ }^{* *} \mathrm{p}<0.01,{ }^{\star * *} \mathrm{p}<0.001$

\section{DISCUSSION}

This study supports earlier findings of increase in capillarity of myocardium in response to exercise and ACE inhibitor treatment in hypertensive rats.

Volume fraction of muscle was decreased in lisinopril and lisinopril + exercise groups as compared to control group. However, since exercise did not change $V_{f}$ of muscle when compared to control, this suggests that the effect on muscle $V_{f}$ is mainly due to lisinopril. Chronic treatment with ACE inhibitor cilazapril has been shown to reduced cardiac hypertrophy and improved coronary vascular reserve in the left and right ventricles (Clozel et al. 1989).

Hypertrophy in hypertensive rats is thought to be due to excessive laying down of myocardial fibrous tissue by rapidly dividing fibroblasts. The mechanism of reduction in muscle mass in myocardium by ACE inhibitor treatment is thought to be due to inhibition of myocardial fibrosis thereby reversing hypertrophy (Crisman et al 1985). The effect of exercise on muscle mass in hypertension is controversial with various studies indicating both hypertrophy as a result of exercise (Barnard, et al. 1980) and a reduction in hypertrophy (Hinderliter, et al. 2002). There were others however, indicating no significant change in muscle mass due to exercise (Bersohn and Scheuer 1977). Our findings here tend to agree with the latter observation. It is thought that the effect of exercise on myocardial mass is mediated via the influence of exercise on collagen transcription (Contard, 1991; Thomas, et al. 2000;). Furthermore, the fact that there was no difference in $V_{f}$ of muscle between lisinopril and lisinopril + exercise groups in this study indicated that the effect on muscle is largely due to lisinopril and not exercise.

(a)

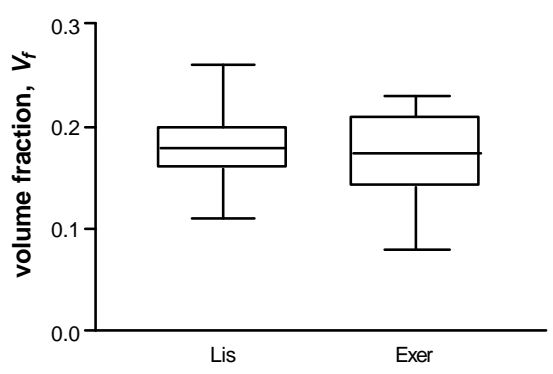

(b)
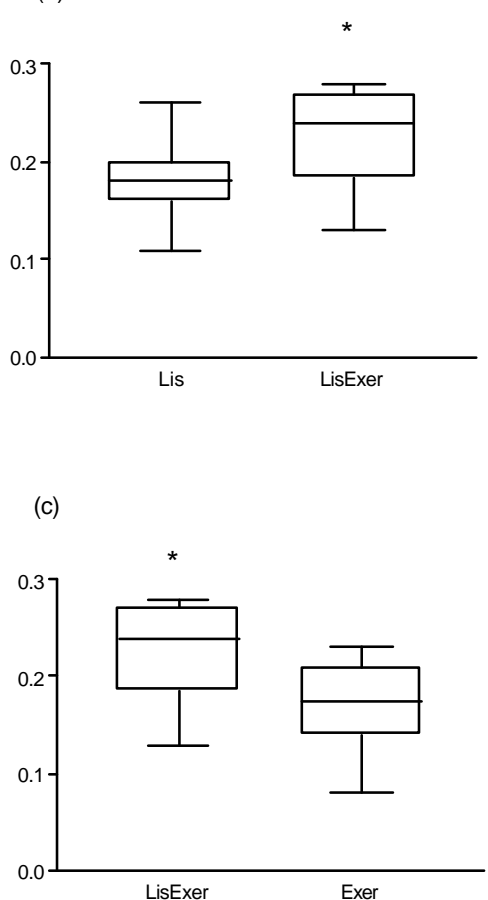

Figure 4

Box plot comparison of $V_{f}$ capillaries between intervention groups. (a) between Lis and Exer, (b) between Lis and LisExer, (c) between LisExerand Exer.

${ }^{\star} p<0.05,{ }^{\star \star} p<0.01,{ }^{\star \star \star} p<0.001$ 
Volume fraction of capillaries showed significant increase in lisinopril, exercise and lisinopril + exercise groups as compared to control indicating that both exercise and lisinopril stimulate capillary growth. It could well be possible however, that the increase in $V_{f}$ capillaries seen in lisinopril is a relative outcome to a decrease in $V_{f}$ muscle and not an actual increase in vessels. This therefore produces a net effect of an increase of $V_{f}$ capillaries. Indeed it was reported by Black et al (2001) that ACE inhibitor treatment enhances vascularization in the adolescent heart through reductions in myocardial mass, but not capillary growth.

However, in the exercise group since there was no significant change in the $V_{f}$ muscle between it and control, this indicates that the increase in $V_{f}$ cap is due to a real increase in capillaries. The mechanism of enhanced capillarity in exercise is thought to involve the bradycardia caused by exercise, which increases myocardial perfusion during diastole.

This in return increases blood flow thereby stimulating capillary growth. Not surprisingly since both ACE inhibitors and exercise cause an increase in $V_{f}$ capillaries of similar magnitude as compared to sedentary controls, there was no significant difference between the two. However, due to this effect on capillaries the $V_{f}$ of interstitial tissue space was slightly increased though not significantly. When lisinopril and exercise were combined together, they appear to potentiate one another leading to higher $V_{f}$ of capillaries than either alone.

In conclusion, it appears from this study that combining moderate exercise with ACE inhibitor lisinopril resulted in a better $V_{f}$ of capillaries and reduction of $V_{f}$ muscle than either of the two alone. The outcome of these changes may well be an enhanced cardiac performance in hypertension.

\section{Acknowledgement}

We would like to thank the Sultan Qaboos University for funding this project. We also gratefully acknowledge the technical assistance of $M r \quad K$ Tahlilkar with the animal experiments.

\section{REFEREN CES}

Anversa P, Levicky V, Beghi C, McDonald S.L, and Kikkawa Y (1983). Morphometry of exercise induced right ventricular hypertrophy in the rat. Circ. Res. 52, 57-64.

Barnard, R.J. Duncan, H.W, Baldwin, K.M, Grimditch, G Buckberg, G.D (1980). "Effects of intensive exercise training on myocardial performance and coronary blood flow." Journal of Applied Physiology: Respiratory, Environmental and Exercise Physiology 49,3: 444-9.

Bersohn, M.M. and J. Scheuer (1977). "Effects of physical training on end-diastolic volume and myocardial performance of isolated rat hearts." Circulation Research (Online) 40,5: 510-6.

Black M.J, Bertram J.F, Johnston C.I (2001). Effect of angiotensin-converting enzyme inhibition on myocardial vascularization in the adolescent and adult spontaneously hypertensive rat. J Hypertension 19, 4: 785-94

Clozel J.P, Kuhn H, and Hefti F (1989). Effects of chronic ACE inhibition on cardiac hypertrophy and coronary vascular reserve in spontaneously hypertensive rats with developed hypertension. J Hypertens 7, 267-275.

Crisman R.P, and Tomanek R.J (1985). Exercise training modifies myocardial mitochondria and myofibril growth in spontaneously hypertensive rats. Am J Physiol 248, H8-14.

Der_Sarkissian S, Mar chand E.L, Duguay D., Hamet P, and deBlois D (2003). Reversal of interstitial fibroblast hyperplasia via apoptosis in hypertensive rat heart with valsartan or enalapril. Cardiovasc Res 57, 775-783.

Heringer-Walther S, Batista E.N, Walther T, Khosla M.C, Santos R.A.S, and Campagnole-Santos MJ (2001). Baroreflex Improvement in SHR After ACE Inhibition Involves Angiotensin. Hypertension 37, 1309-1314.

Himeno E, Nishino K, Nakashima Y, Kuroiwa A, and Ikeda M (1996). Weight reduction regresses left ventricular mass regardless of blood pressure level in obese subjects. Am Heart J 131, 313-319.

Hinderliter A, Sherwood A, Gullette E.C, Babyak M, Waugh R, Georgiades A, and Blumenthal JA (2002). Reduction of left ventricular hypertrophy after exercise and weight loss in overweight patients with mild hypertension. Arch Intern Med 162, 1333-1339.

Lazar H.L, Bao Y, Rivers S, Colton T, and Bernard S.A. (2001). High tissue affinity angiotensin converting enzyme inhibitors improve endothelial function and reduce infarct size. Ann Thorac Surg 72, 548-553.

Mattfeldt T, Mall G, Garehbaghi H, and Moller P. (1990). Estimation of surface area and length with the orientator. J. Microsc. 159, 301-317

Zimmerman T.D.S, et al. (2000). "Collagen gene expression in rat left ventricle: interactive effect of age and exercise training." J. Appl. Physiol 89, 4: 1462-8.

Address for Correspondence:

Dr Ibrahim M Inuwa, Department of Human and Clinical Anatomy, Sultan Qaboos University, PO Box 35. Al Khod 123. Muscat, Sultanate of Oman. Tele phone: +968 515176, Facsimile: +968513419 , Email: ibrahim1@squ.edu.om 